\title{
Hospitalização por Condições Sensíveis à Atenção Primária em menores de cinco anos de idade em Santa Catarina, 2012: estudo descritivo*
}

doi: $10.5123 /$ S1679-49742018000300006

\author{
Hospitalization for Ambulatory Care Sensitive Conditions in children under five years old in \\ Santa Catarina State, Brazil, 2012: a descriptive study
}

Hospitalización por Condiciones Sensibles a la Atención Primaria en niños de Santa Catarina, Brasil, 2012: estudio descriptivo

\author{
Tatiana da Silva Oliveira Mariano' 1 - (1) orcid.org/0000-0002-8763-4385 \\ Fúlvio Borges Nedel ${ }^{2,3}$
}

'Universidade Federal de Santa Catarina, Programa de Pós-Graduação em Saúde Coletiva, Florianópolis, SC, Brasil

¿Universidade Federal de Santa Catarina, Centro de Ciências da Saúde, Departamento de Saúde Pública, Florianópolis, SC, Brasil

${ }^{3}$ Universitat Autònoma de Barcelona, Unitat de Bioestadística, Grups de Recerca d'Amèrica i Àfrica Llatines, Barcelona, Espanha

\section{Resumo}

Objetivo: descrever as proporções e taxas de hospitalização por Condições Sensíveis à Atenção Primária (CSAP) em menores de 5 anos de idade no estado de Santa Catarina, Brasil, em 2012. Métodos: estudo descritivo, com dados do Sistema de Informações Hospitalares do Sistema Único de Saúde (SIH/SUS), classificados segundo a Lista Brasileira de CSAP. Resultados: foram registradas 32.445 hospitalizações em $<5$ anos de idade; as CSAP representaram $25,7 \%$ delas, com taxa de 20,1/1.000 habitantes do mesmo grupo etário; as principais causas foram gastroenterites infecciosas e complicações $(26,7 \%)$, pneumonias bacterianas $(22,2 \%)$ e doenças pulmonares $(16,9 \%)$; houve maior taxa de hospitalização por CSAP no sexo masculino (21,1/1.000) e em menores de 1 ano de idade (43,8/1.000). Conclusão: os resultados evidenciaram que o estado de Santa Catarina apresentou taxas e proporções inferiores às observadas em outros estudos brasileiros, embora as CSAP tenham sido causa de um quarto das internações em $<5$ anos.

Palavras-chave: Atenção Primária à Saúde; Epidemiologia Descritiva; Hospitalização; Pediatria; Indicadores Básicos de Saúde.

\footnotetext{
*Este artigo compõe a dissertação de mestrado acadêmico em Saúde Coletiva intitulada 'Hospitalização por Condições Sensíveis à Atenção Primária em menores de cinco anos de idade em Santa Catarina, 2012', defendida por Tatiana da Silva Oliveira Mariano junto ao Programa de Pós-Graduação em Saúde Coletiva da Universidade Federal de Santa Catarina (UFSC) no ano de 2017, com apoio de bolsa de mestrado disponibilizada pela Coordenação de Aperfeiçoamento de Pessoal de Nível Superior (Capes) do Ministério da Educação.
}

\footnotetext{
Endereço para correspondência:
}

Tatiana da Silva Oliveira Mariano - Universidade Federal de Santa Catarina, Centro de Ciências da Saúde, Programa de PósGraduação em Saúde Coletiva, Campus Reitor João David Ferreira Lima, Rua Delfino Conti, s/n, Bloco H, Florianópolis, SC, Brasil. CEP: $88040-900$

E-mail: oliveira.tatianasilva@gmail.com 


\section{Introdução}

A efetividade da Atenção Primária à Saúde (APS) é de grande interesse para gestores, profissionais da saúde e cidadãos. Com a intenção de verificar se a APS tem desempenhado seu papel com presteza e efetividade, são necessários instrumentos capazes de avaliá-la de maneira simples e concisa. ${ }^{1} 0$ indicador de internação por Condições Sensiveis à Atenção Primária (CSAP) tem sido um desses instrumentos, utilizado na medição da capacidade de resolução de problemas de saúde pela primeira instância de atenção de um sistema de saúde. ${ }^{2}$ Data do início dos anos 1990 a definição das CSAP como condições de saúde de interesse para uma APS oportuna e efetiva, capaz de reduzir o risco de hospitalização pela prevenção de agravos, controle de episódios agudos e manejo de doenças crônicas. ${ }^{3}$

Em 2005, iniciou-se o processo de construção da Lista Brasileira de Internações por CSAP com 0 levantamento das correspondentes listas nacionais e estrangeiras, reuniões para elaboração e revisão, validação pela Sociedade Brasileira de Medicina de Família e Comunidade e, entre outubro e novembro de 2007, sua disponibilização para consulta pública. ${ }^{4}$ Finalmente, a Portaria do Ministério da Saúde SAS/ MS $n^{0} 221$, de 17 de abril de 2008, instituiu a Lista Brasileira de Internações por CSAP, composta por 19 grupos de problemas de saúde. ${ }^{5}$

\section{A descrição das hospitalizações por Condições Sensíveis à Atenção Primária permite aos gestores estabelecer regiões ou municípios prioritários no estabelecimento de políticas para melhor organização da Atenção Primária à Saúde.}

De acordo com revisão bibliográfica realizada por estes autores nas bases de dados Pubmed, Lilacs, Scopus, entre outras, foram encontrados 25 artigos estrangeiros, publicados no período de 1996-2016, e 25 artigos brasileiros, de 2008-2016, que abordaram as hospitalizações por CSAP na população pediátrica em suas análises. ${ }^{6}$ Esses estudos assinalaram diferenças significativas nas taxas de hospitalização por CSAP segundo faixas etárias, com maior prevalência e/ou incidência em crianças mais jovens. ${ }^{6,7}$ Altas taxas de admissão hospitalar por
CSAP em uma área ou subgrupo populacional podem indicar problemas sérios de acesso ou funcionamento do cuidado ambulatorial. ${ }^{8}$ Estudos brasileiros mostram que o risco de hospitalização por CSAP em menores de 5 anos é maior quando comparado ao de indivíduos de 5 a 59 anos de idade. ${ }^{9} 0$ evidente protagonismo das altas taxas de internação hospitalar por CSAP em menores de 5 anos estimula seu estudo nessa população. ${ }^{10,11}$ Não foram encontrados trabalhos de pesquisa no estado de Santa Catarina sobre admissões hospitalares por CSAP na população pediátrica, que utilizassem todos os códigos da Lista Brasileira de Internações por CSAP. A descrição das hospitalizações por CSAP permite aos gestores estabelecer regiões ou municípios prioritários no estabelecimento de políticas para melhor organização da Atenção Primária à Saúde.

0 presente estudo teve por objetivo descrever as proporções e taxas de hospitalização por CSAP em menores de 5 anos de idade no estado de Santa Catarina, Brasil, em 2012.

\section{Métodos}

Trata-se de um estudo descritivo sobre as proporções e as taxas de hospitalização por CSAP em menores de 5 anos de idade residentes no estado de Santa Catarina em 2012.

Segundo dados do Censo de 2010, Santa Catarina abrigava 6.248.436 habitantes, $84 \%$ deles vivendo em áreas urbanas. ${ }^{12}$ Cerca de $80 \%$ dos 293 municípios possuíam menos de 20 mil habitantes. A proporção de crianças menores de 5 anos de idade diminuiu de cerca de $11,2 \%$ do total populacional em 1990 , para $6,5 \%$ em 2010. ${ }^{12} 0$ estado apresenta nove macrorregiões de saúde (Extremo Oeste, Meio Oeste, Planalto Norte, Nordeste, Vale do Rio Itajaí, Foz do Rio Itajaí, Grande Florianópolis, Planalto Serrano e Sul), estruturadas e organizadas para atender à demanda de atendimentos de média e alta complexidade. ${ }^{13}$

A totalidade de hospitalizações, por município de residência, e as características epidemiológicas analisadas foram obtidas das Autorizações de Internação Hospitalar (AIH) pagas e registradas no Sistema de Informações Hospitalares do Sistema Único de Saúde (SIH/SUS), disponibilizadas no sítio eletrônico do Departamento de Informática do SUS (Datasus). Foram incluídas AIH de pacientes admitidos em 2012, embora contabilizadas em 2013: por exemplo, no banco de 
maio de 2013 ainda constavam AIH de pessoas admitidas por unidades hospitalares-SUS em dezembro de 2012. Fatos como esse ocorrem devido a erros ou inconsistências no preenchimento da AIH, a qual retorna ao profissional que a assinou para correção. Este procedimento, somado ao tempo de retorno e envio ao Ministério da Saúde, pode demorar meses, razão por que a busca das AIH foi estendida até junho de 2013. Cumpre reafirmar que foram analisadas somente as admissões hospitalares de menores de 5 anos de idade residentes no estado de Santa Catarina entre $1^{\circ}$ de janeiro e 31 de dezembro de 2012.

A classificação como Condição Sensível à Atenção Primária seguiu a Lista Brasileira de Internações por CSAP, instituída pelo Ministério da Saúde em 2008. Essa lista é composta por 19 grupos de causas de hospitalização, com base na Classificação Estatística Internacional de Doenças e Problemas Relacionados à Saúde - 10 $0^{\text {a }}$ Revisão (CID-10). ${ }^{5}$

Foi realizado teste de consistência dos dados para verificação do número de hospitalizações por CSAP em menores de 5 anos de idade entre 2007 e 2012. 0 teste de consistência tem a finalidade de identificar discrepâncias no número de hospitalizações contabilizadas, alertando para possíveis erros no sistema ou fraudes; por isso foi escolhido o período de 5 anos anteriores a a ano estudado. Caso a diferença entre 0 número de admissões hospitalares por CSAP faturadas sobre um determinado ano fosse maior que o dobro do ano anterior, seria feita a análise por cada mês daquele ano. Como o ano considerado para estudo foi 2012, tão somente, houve análise mensal deste ano. Constatou-se que os municípios Anchieta (6.172 hab.), Braço do Trombudo (3.498 hab.), Lageado Grande (1.478 hab.), Mirim Doce (2.477 hab.), Palmeira (2.410 hab.), Passo de Torres (6.964 hab.) e Presidente Nereu (2.281 hab.) não registraram nenhuma internação por CSAP em menores de 5 anos de idade, no ano de 2012. Contudo, crianças catarinenses menores de 5 anos podem ter sido admitidas em hospitais de outros estados, visto que a busca se limitou às hospitalizações em Santa Catarina.

0 tamanho da população residente na faixa etária estudada foi obtido de estimativa populacional para 2012, com base no Censo de 2010 da Fundação Instituto Brasileiro de Geografia e Estatística (IBGE). Na descrição das hospitalizações, foram calculadas as proporções pelo quociente entre o número de hospitalizações por
CSAP e o total de hospitalizações, multiplicado por 100; e as taxas, pelo quociente entre o número de hospitalizações por CSAP e o total de habitantes, multiplicado por 1.000. Para os resultados globais, o numerador e o denominador corresponderam ao total das crianças com até 5 anos de idade; e para resultados específicos, o numerador e o denominador corresponderam (i) ao sexo (masculino; feminino), (ii) à faixa etária (menor de um ano e 1 a 4 anos de idade), (iii) aos grupos de causas de internação (gastroenterites infecciosas e complicações; pneumonias bacterianas; doenças pulmonares; asma; infecção no rim e no trato urinário; epilepsias; infecção de pele e subcutânea; infecções de ouvido, nariz e garganta; doenças preveníveis por imunização e condições sensíveis; deficiências nutricionais; doenças relacionadas ao pré-natal e parto; insuficiência cardíaca; diabetes mellitus; anemia; úlcera gastrointestinal; doenças cerebrovasculares; hipertensão; doença inflamatória de órgãos pélvicos femininos; e angina) e (iv) macrorregiões de saúde.

As taxas de hospitalização foram padronizadas por sexo e faixa etária, pelo método indireto. Como a correlação entre a razão de hospitalização padronizada e as taxas brutas foi alta (coeficiente de correlação de Pearson=0,99), considerou-se desnecessária a padronização e analisou-se a taxa bruta.

Os arquivos das AIH reduzidas foram expandidos do formato .DBC para .DBF, pelo programa TabWin; o processamento dos dados e as análises estatísticas foram feitos pelo software Stata versão 14.2; e o mapa de distribuição geográfica das taxas, desenhado pelo programa R, com auxílio dos pacotes tmap e tmpatools.

As informações utilizadas são de domínio público, sem identificação individual. 0 projeto do estudo, em conformidade com a Resolução do Conselho Nacional de Saúde (CNS) n ${ }^{\circ}$ 466, de 12 de dezembro de 2012, prescindiu de apreciação por Comitê de Ética em Pesquisa.

\section{Resultados}

Referentes ao ano de 2012, foram registradas 32.445 hospitalizações em menores de 5 anos de idade residentes no estado de Santa Catarina (78,2 por 1.000 habitantes); 25,7\% dessas hospitalizações foram por CSAP (20,1 por 1.000 hab.) (Tabela 1). Na Figura 1, observa-se a distribuição geográfica das taxas de hospitalização por CSAP em menores de 5 anos, majoritariamente concentradas na região central do estado. 
Os três principais grupos de causas de hospitalização por CSAP identificados foram gastroenterites infecciosas e complicações $(26,7 \%)$, pneumonias bacterianas $(22,2 \%)$ e doenças pulmonares (16,9\%); este conjunto corresponde, aproximadamente, a dois terços das hospitalizações por CSAP em menores de 5 anos de idade em Santa Catarina. A proporção de gastroenterites infecciosas e complicações foi cerca de 20,0\% maior em relação à de pneumonias bacterianas, entre os internados por CSAP (Tabela 1).

A proporção de hospitalizações por CSAP foi 12,0\% maior em crianças do sexo feminino, comparadas às do sexo masculino; para crianças entre 1 e 4 anos de idade, essa proporção foi $25 \%$ maior, relativamente à de menores de 1 ano.

A macrorregião do Meio Oeste apresentou maior proporção e maior taxa de hospitalização por CSAP, sendo essa proporção 2,8 vezes maior e a taxa de hospitalização 3,5 vezes maior que as correspondentes para Foz do Rio Itajaí, macrorregião de saúde onde foram observados os menores valores do estado. 0 Planalto Serrano apresentou a segunda maior taxa e a terceira maior proporção de hospitalizações por CSAP, enquanto a macrorregião Sul referiu a terceira maior taxa e a segunda maior proporção correspondentes (Tabela 2).

A taxa de hospitalização por CSAP em crianças do sexo masculino foi cerca de $11,0 \%$ maior $(21,1$ por 1.000 hab.) em relação à de crianças do sexo feminino (19,1 por 1.000 hab.). Quanto à faixa etária, a taxa de hospitalização por CSAP foi aproximadamente três vezes maior em crianças menores de 1 ano, em relação à de crianças de $1 \mathrm{a} 4$ anos de idade (Tabela 2).

\section{Tabela 1 - Número de hospitalizações em menores de 5 anos de idade, proporções e taxas de hospitalização por Condições Sensíveis à Atenção Primária (por 1.000 habitantes), segundo grupo de causas e demais causas de hospitalizações, Santa Catarina, 2012}

\begin{tabular}{|c|c|c|c|c|}
\hline Grupo de causas de hospitalização por CSAPa,b & N & $\begin{array}{c}\text { Total } \\
\% \\
\end{array}$ & $\begin{array}{c}\text { CSAP } \\
\%\end{array}$ & Taxa \\
\hline Gastroenterites infecciosas e complicações & 2.230 & 6,87 & 26,72 & 5,38 \\
\hline Pneumonias bacterianas & 1.854 & 5,71 & 22,21 & 4,47 \\
\hline Doenças pulmonares & 1.413 & 4,36 & 16,93 & 3,41 \\
\hline Asma & 663 & 2,04 & 7,94 & 1,60 \\
\hline Infecção no rim e no trato urinário & 548 & 1,69 & 6,57 & 1,32 \\
\hline Epilepsias & 434 & 1,34 & 5,20 & 1,05 \\
\hline Infecção de pele e subcutânea & 378 & 1,17 & 4,53 & 0,91 \\
\hline Infecções de ouvido, nariz e garganta & 328 & 1,01 & 3,93 & 0,79 \\
\hline Doenças preveníveis por imunização e condições sensíveis & 214 & 0,66 & 2,56 & 0,52 \\
\hline Deficiências nutricionais & 130 & 0,40 & 1,56 & 0,31 \\
\hline Doenças relacionadas ao pré-natal e parto & 39 & 0,12 & 0,47 & 0,09 \\
\hline Insuficiência cardíaca & 36 & 0,11 & 0,43 & 0,09 \\
\hline Diabetes mellitus & 31 & 0,10 & 0,37 & 0,07 \\
\hline Anemia & 19 & 0,06 & 0,23 & 0,05 \\
\hline Úlcera gastrointestinal & 13 & 0,04 & 0,16 & 0,03 \\
\hline Doenças cerebrovasculares & 10 & 0,03 & 0,12 & 0,02 \\
\hline Hipertensão & 4 & 0,01 & 0,05 & 0,01 \\
\hline Doença inflamatória de órgãos pélvicos femininos & 2 & 0,01 & 0,02 & 0,00 \\
\hline Angina & 1 & - & 0,01 & - \\
\hline Total por CSAP a & 8.347 & 25,73 & 100,00 & 20,13 \\
\hline Demais causas & 24.098 & 74,27 & - & 58,12 \\
\hline Total & 32.445 & 100,00 & - & 78,25 \\
\hline
\end{tabular}

a) CSAP:Condições Sensíveis à Atenção Primária.

b) Portaria SAS/MS n 221 , de 17 de abril de $2008^{5}$ 


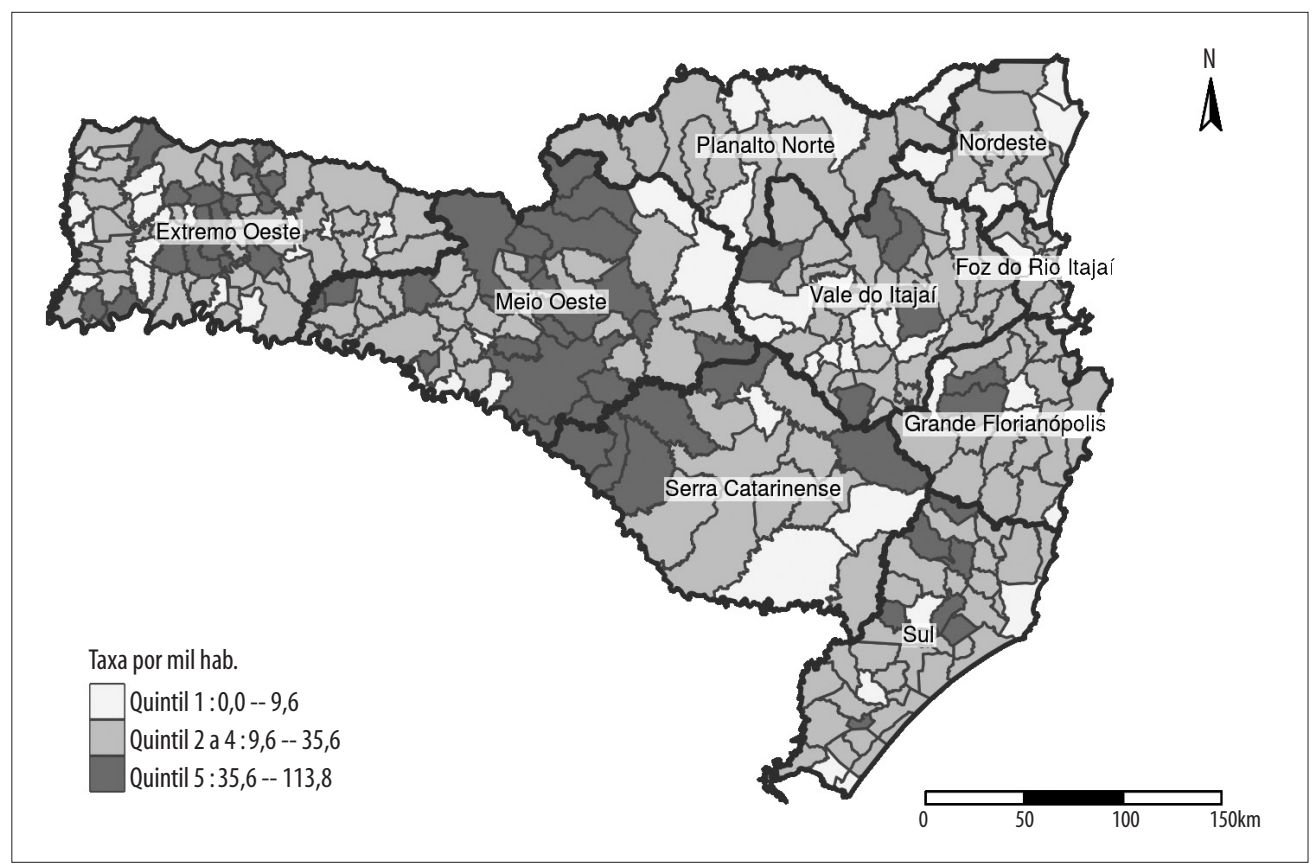

Nota: em destaque, circunscritas por linhas mais grossas, as macrorregiões de saúde de Santa Catarina.

\section{Figura 1 - Distribuição geográfica das taxas de hospitalização por Condições Sensíveis à Atenção Primária em menores de 5 anos de idade (por 1.000 habitantes), por municípios, Santa Catarina, 2012}

\section{Discussão}

Em Santa Catarina, no ano de 2012, aproximadamente uma a cada 50 crianças residentes no estado foi internada em hospitais por causas que poderiam ser evitadas se houvesse um melhor funcionamento do sistema de saúde em sua primeira instância de atenção. Considerando-se a capacidade da APS em resolver as necessidades de saúde da população, aproximadamente um quarto das hospitalizações de menores de 5 anos de idade seria evitado se esse cuidado fosse prestado de forma efetiva, no momento oportuno. A taxa de hospitalização foi maior em crianças do sexo masculino, menores de 1 ano e residentes na macrorregião Meio Oeste. As principais causas de hospitalização por CSAP em menores de 5 anos de idade foram gastroenterites infecciosas e complicações, pneumonias bacterianas e doenças pulmonares.

Comparada com os estados do Piauí (61/1.000 hab. em 2010), Espírito Santo (31/1.000 hab. em 2009) e Pernambuco (62/1.000 hab. em 2009), Santa Catarina apresentou menor taxa de hospitalização por CSAP em menores de 5 anos. ${ }^{14-16}$ Estudo realizado na Carolina do Sul, Estados Unidos, encontrou uma taxa de hospitalização de 25/1.000 hab. em $1995 .{ }^{10}$ Uma análise das hospitalizações ocorridas em 2010 no Equador, utilizando-se da lista brasileira de CSAP, evidenciou maior risco de hospitalização em menores de 1 ano, relativamente à faixa etária de 1 a 4 anos. ${ }^{17}$ No presente estudo, a proporção de CSAP entre hospitalizações de crianças de 1 a 4 anos foi $25 \%$ maior em relação às hospitalizações de menores de 1 ano. A taxa de internação hospitalar entre crianças menores de 1 ano foi três vezes maior que a da faixa de 1-4 anos, semelhantemente ao encontrado em outros locais do Brasil..$^{14,18,19}$ Em Valência, Espanha, crianças entre 2 e 4 anos de idade apresentaram odds ratio de 0,50 (0,29-0,84), em relação àquelas com menos de um ano de vida. ${ }^{20}$ Para estas crianças, a maior taxa de hospitalização seria justificada pela maior susceptibilidade a infecções e maior tendência de encaminhamento para internação hospitalar pelos profissionais..$^{20}$ Sem a adequada abordagem na APS, essas crianças estariam mais 
Tabela 2 - Frequências absoluta e relativa e taxas de hospitalização por Condições Sensíveis à Atenção Primária (por 1.000 habitantes menores de 5 anos de idade), segundo sexo, faixa etária e macrorregião de saúde, Santa Catarina, 2012

\begin{tabular}{lcccc}
\hline Variável & N & $\%$ & $\begin{array}{c}\text { CSAPa } \\
\%\end{array}$ & Taxa \\
\hline Sexo & & & & 19,10 \\
\hline Feminino & 3.881 & 46,50 & 27,37 & 21,12 \\
Masculino & 4.466 & 53,50 & 24,45 & 43,76 \\
\hline Faixa etária (em anos) & & & 22,92 & 13,88 \\
\hline$<1$ & 3.795 & 45,47 & 28,65 & 37,10 \\
$1-4$ & 4.552 & 54,53 & & 25,60 \\
\hline Macrorregião de saúde & & & 37,14 & 17,71 \\
\hline Meio Oeste & 1.510 & 18,09 & 32,07 & 17,03 \\
Sul & 1.496 & 17,92 & 21,96 & 20,15 \\
Vale do Itajaí & 1.067 & 12,78 & 23,38 & 14,48 \\
Nordeste & 1.038 & 12,44 & 22,57 & 30,24 \\
Extremo Oeste & 952 & 11,41 & 26,27 & 10,63 \\
Grande Florianópolis & 900 & 10,78 & 29,95 & 14,35 \\
Planalto Serrano & 596 & 7,14 & 13,39 & 20,13 \\
Foz do Rio Itajaí & 421 & 5,04 & 22,39 & 25,73 \\
Planalto Norte & 367 & 4,40 & & \\
\hline Total & 8.347 & 100,00 & & \\
\hline
\end{tabular}

a) CSAP: Condições Sensiveis à Atenção Primária.

sujeitas às danosas consequências de possíveis falhas no sistema. Na literatura, o sexo masculino associa-se a maior probabilidade de hospitalização por CSAP na população pediátrica. ${ }^{10,21}$ Estudo com base na Pesquisa Nacional de Demografia e Saúde da Criança e da Mulher (PNDS/2006), ${ }^{21}$ sobre menores de 2 anos de idade hospitalizados por CSAP no ano anterior, encontrou uma razão de prevalências de $1,47(\mathrm{p}=0,004)$ para o sexo masculino; em Valência, porém, a odds ratio de hospitalização por CSAP em meninos foi de $0,60(0,41-0,89)$ para a população menor de 15 anos de idade. ${ }^{20}$ No presente estudo, 0 sexo masculino apresentou taxa $11 \%$ maior, embora a proporção de CSAP entre as internações tenha sido 11\% menor nos meninos. Na Carolina do Sul, as hospitalizações por CSAP em menores de 18 anos de idade registraram uma proporção cerca de $4 \%$ menor para o sexo masculino. ${ }^{10}$

Na macrorregião de saúde do Meio Oeste houve maior proporção de diagnósticos de CSAP entre as hospitalizações, e maior taxa de hospitalização por CSAP. A justificativa para esse achado pode estar na associação entre altas taxas de hospitalização por CSAP e menor porte populacional. ${ }^{22,23}$ A macrorregião do Meio Oeste catarinense apresenta mediana de 4.632 hab. entre seus municípios (maior apenas que a macrorregião de saúde do Extremo Oeste, com 4.328,5 hab.), o que, por sua vez, pode estar relacionado à chamada "lei de Roemer" - "Se há leitos hospitalares, eles tendem a ser usados." -, que sinaliza o uso dos leitos hospitalares em ociosidade independentemente da necessidade da população. ${ }^{24}$ Um estudo de 2010 sobre a associação entre disponibilidade de leitos hospitalares e taxas de hospitalização no estado de Michigan, Estados Unidos, constitui uma forte evidência da "lei de Roemer" ao demonstrar associação positiva consistente entre os níveis de agregação para taxas de hospitalização por CSAP, infarto do miocárdio, acidente vascular cerebral e fratura pélvica, cobertura de plano de saúde, proporçãa de afro-americanos, elevadas renda e educação, e maior morbidade..$^{24}$ Outras possíveis justificativas para esse achado podem estar relacionadas a características econômicas, 
político-administrativas, estruturais, de processo de trabalho em saúde, entre outras. ${ }^{2,9,23,25}$

A pneumonia bacteriana é a principal causa de internação por CSAP no país. ${ }^{9,25}$ No estado norte-americano da Carolina do Sul, a principal causa de hospitalização por CSAP foi pneumonia bacteriana em menores de 18 anos de idade, com taxa de 2/1.000 hab. para os residentes em áreas mais ricas e de 5/1.000 hab. para os mais pobres. ${ }^{10}$ Em menores de 5 anos de idade, o grupo de causas 'Gastroenterites infecciosas e complicações' se apresenta como o principal problema de hospitalização por CSAP no Brasil, ${ }^{26}$ em consonância com os resultados deste estudo e os encontrados para os estados do Piauí, Espírito Santo e Pernambuco. ${ }^{14-16}$ No Equador, as gastroenterites foram a principal causa de hospitalização por CSAP no país, e representaram $65 \%$ das CSAP em menores de 5 anos. ${ }^{17}$ A ocorrência de gastroenterites infecciosas e complicações está associada à infraestrutura sanitária do local de moradia da família, enquanto a hospitalização por essas causas indica abordagem tardia de crianças desidratadas, ou seja, uma falha da APS. ${ }^{21}$

Um fator limitador para as análises deste estudo está no fato de somente contabilizar dados de internações em unidades geridas ou conveniadas ao SUS. Apesar disto, 70\% dos catarinenses se internam pelo SUS, ${ }^{27}$ permitindo que os resultados observados sejam aplicados à gestão da APS no estado. Outro ponto a se discutir remete à qualidade da informação disponível. Pode-se admitir, e até enfatizar, entre os maiores problemas da confiabilidade, a possibilidade de fraude visando aumentar 0 reembolso financeiro por internações. Contudo, desde a criação do SUS, houve um reconhecido avanço nas auditorias do sistema como prática regular, o que minimizou esse problema. ${ }^{28}$ Estudo sobre confiabilidade do diagnóstico de CSAP nos registros do SIH/SUS concluiu por sua validade, tendo encontrado concordância substancial a quase perfeita $(\mathrm{k}=0,784)$, sensibilidade de $78,2 \%$ e especificidade de $97 \%$ entre as AIH e os prontuários hospitalares. ${ }^{29}$ Outra limitação foi a utilização de AIH somente do estado de Santa Catarina, sem contabilizar admissões hospitalares por CSAP de menores de 5 anos de idade residentes em Santa Catarina mas ocorridas em outros estados do país. Supõe-se que estas internações não sejam significativas para o estudo, dado o total de hospitalizações analisadas.

0 fundamento do indicador de internações por CSAP reside na elevada capacidade resolutiva da APS, atribuível a medidas de promoção de saúde, preventivas e de tratamento ambulatorial, que deveriam implicar uma diminuição das hospitalizações em função de detecção precoce e controle de enfermidades em estágios menos avançados. ${ }^{2,30}$

0 presente estudo contribuiu para o conhecimento do perfil das hospitalizações por CSAP em menores de 5 anos de idade no estado de Santa Catarina, no ano de 2012. 0 estado exibiu a menor taxa de hospitalização, comparado a três outros estados do país, analisados por outros estudos. Entre as crianças internadas, houve maior proporção de diagnósticos de CSAP nas meninas e crianças de 1 a 4 anos de idade. Observou-se maior taxa de hospitalização por CSAP entre crianças do sexo masculino e em menores de um ano de idade. 0 principal grupo de causas de CSAP foi o das gastroenterites infecciosas, em concordância com outros estudos sobre o tema, o que sugere investigações quanto à cobertura de saneamento básico, distribuição do soro de reidratação oral e oportunidade da abordagem terapêutica de crianças em desidratação. 0 Meio Oeste catarinense apresentou as maiores proporções e taxas de hospitalização por CSAP em menores de 5 anos de idade, possivelmente um resultado da alta concentração de municípios de pequeno porte nessa macrorregião de saúde. Tais informações a respeito das características epidemiológicas das internações hospitalares por CSAP no grupo pediátrico podem auxiliar no planejamento de ações específicas e resolutivas, pela melhor Atenção Primária à Saúde nos municípios do estado de Santa Catarina.

\section{Contribuição dos autores}

Mariano TSO realizou a aquisição, análise e interpretação dos dados, e redigiu as versões preliminares do manuscrito. Nedel FB foi responsável pela concepção do estudo, colaborou na aquisição, análise e interpretação dos dados e na revisão crítica de todas as versões do manuscrito. Ambos os autores concordam com a versão final e se responsabilizam por todos os aspectos do trabalho, incluindo a garantia de sua precisão e integridade. 


\section{Referências}

1. Starfield B. Atenção primária: equilíbrio entre necessidades de saúde, serviços e tecnologia [Internet]. Brasília: UNESCO, Ministério da Saúde; 2002 [citado 2018 mar 5]. 726 p. Disponível em: https://www.nescon.medicina.ufmg.br/biblioteca/ imagem/0253.pdf

2. Nedel FB, Facchini LA, Bastos JL, Martín-Mateo M. Conceptual and methodological aspects in the study of hospitalizations for ambulatory care sensitive conditions. Ciênc Saúde Coletiva. 2011;16 Suppl 1:1145-54.

3. Billings J, Zeitel L, Lukomnik J, Carey TS, Blank $\mathrm{AE}$, Newman L. Impact of socioeconomic status on hospital use in New York city. Health Aff (Millwood). 1993 Spring; 12(1):162-73

4. Alfradique ME, Bonolo PF, Dourado I, Lima-Costa MF, Macinko J, Mendonça CS, et al. Internações por condições sensíveis à atenção primária: a construção da lista brasileira como ferramenta para medir o desempenho do sistema de saúde (projeto ICSAP - Brasil). Cad Saúde Pública. 2009 jun;25(6):1337-49.

5. Brasil. Ministério da Saúde. Portaria n 221, de 17 de abril de 2008. Publica na forma de anexo desta portaria, a lista brasileira de internações por condições sensíveis à atenção primária. Diário Oficial da República Federativa do Brasil, Brasília (DF), 2008 abr 18; Seção 1:70.

6. Mariano TSO. Hospitalização por condições sensíveis à atenção primária em menores de cinco anos de idade em Santa Catarina, 2012 [dissertação]. Florianópolis (SC): Universidade Federal de Santa Catarina; 2017.

7. Pereira FJR, Silva CC, Lima Neto EA. Condições sensíveis à atenção primária: uma revisão descritiva dos resultados da produção acadêmica brasileira. Saúde Debate. 2014 out;38(esp):331-42.

8. Billings J, Anderson GM, Newman LS. Recent findings on preventable hospitalizations. Health Aff (Millwood). 1996 Fall;15(3):239-49

9. Dourado I, Oliveira VB, Aquino R, Bonolo P, LimaCosta MF, Medina MG, et al. Trends in primary health care-sensitive conditions in Brazil: the role of the family health program (project ICSAP-Brasil). Med Care. 2011 Jun;49(6):577-84

10. Shi L, Samuels ME, Pease M, Bailey WP, Corley EH. Patient characteristics associated with hospitalizations for ambulatory care sensitive conditions in South Carolina. South Med J. 1999 Oct;92(10):989-98.

11. Orueta JF, García-Alvarez A, Grandes G, Nuño-Solinís $\mathrm{R}$. The origin of variation in primary care process and outcome indicators: patients, professionals, centers, and health districts. Medicine (Baltimore). 2015 Aug;94(31):e1314-23.

12. Secretaria Estadual de Saúde (SC). Plano estadual de saúde 2012-2015 [Internet]. Florianópolis: Secretaria Estadual de Saúde; 2011 [citado 2018 mar 5]. 212 p. Disponível em: http://portalses.saude.sc.gov.br/index. php?option $=$ com_docman\&task $=$ doc download\&gid $=5771 \&$ Itemid $=251$

13. Secretaria Estadual de Saúde (SC). PDR 2008 : plano diretor de regionalização [Internet]. Florianópolis: Secretaria Estadual de Saúde; 2008 [citado 2018 mar 5]. 133 p. Disponível em: http://portalses.saude.sc.gov.br/index. php?option $=$ com_docman\&task $=$ doc download\&gid $=2524 \&$ Itemid $=249$

14. Barreto JOM, Nery IS, Costa MSC. Estratégia saúde da família e internações hospitalares em menores de 5 anos no Piauí, Brasil. Cad Saúde Pública. 2012 mar;28(3):515-26.

15. Pazó RG, Frauches DO, Galvêas DP, Stefenoni AV, Cavalcante ELB, Pereira-Silva FH. Internações por condições sensíveis à atenção primária no Espírito Santo: estudo ecológico descritivo no período 2005-2009. Epidemiol Serv Saúde. 2012 abrjun;21(2):275-82.

16. Carvalho SC, Mota E, Dourado I, Aquino R, Teles C, Medina MG. Hospitalizations of children due to primary health care sensitive conditions in Pernambuco state, Northeast, Brazil. Cad Saúde Pública. 2015 abr;31(4):744-54.

17. Nedel FB. Evaluación del impacto de la atención primaria. In: Bedoya R, editor. Medicina familiar: reflexiones desde la práctica [Internet]. Quito (EC): Ministerio de Salud Pública, Organización Panamericana de la Salud; 2017 [citado 2018 mar 5]. p: 303-28. Disponible em: http:// www.paho.org/ecu/index.php?option=com docman\&view $=$ download\&alias $=696$-medicinafamiliar-reflexiones-desde-la-practica\&category_ slug=comunicacion-social\&Itemid=1019 
18. Junqueira RMP, Duarte EC. Internações hospitalares por causas sensíveis à atenção primária no Distrito Federal, 2008. Rev Saúde Pública. 2012 out;46(5):761-8.

19. Santos ILF, Gaíva MAM, Abud SM, Ferreira SMB. Hospitalização de crianças por condições sensíveis à atenção primária. Cogitare Enferm. 2015 janmar;20(1):171-9.

20. Casanova C, Colomer C, Starfield B. Pediatric hospitalization due to ambulatory care-sensitive conditions in Valencia (Spain). Int J Qual Health Care. 1996 Feb;8(1):51-9.

21. Konstantyner T, Mais LA, Taddei JAAC. Factors associated with avoidable hospitalisation of children younger than 2 years old: the 2006 brazilian national demographic health survey. Int J Equity Health. 2015 Aug;14:69.

22. Costa JSD, Büttenbender DC, Hoefel AL, Souza LL. Hospitalizações por condições sensíveis à atenção primária nos municípios em gestão plena do sistema. Cad Saúde Pública. 2010 fev;26(2):358-64.

23. Pazó RG, Frauches DO, Molina MCB, Cade NV. Modelagem hierárquica de determinantes associados a internações por condições sensíveis à atenção primária no Espírito Santo, Brasil. Cad Saúde Pública. 2014 set;30(9):1891-902.

24. Delamater PL, Messina JP, Grady SC, WinklerPrins V, Shortridge AM. Do more hospital beds lead to higher hospitalization rates? A spatial examination of Roemer's law. PLoS One. 2013 Feb;8(2):e54900.
25. Boing AF, Vicenzi RB, Magajewski F, Boing AC, Moretti-Pires R0, Peres KG, et al. Redução das internações por condições sensíveis à atenção primária no Brasil entre 1998-2009. Rev Saúde Pública. 2012 abr;46(2):359-66.

26. Moura BLA, Cunha RC, Aquino R, Medina MG, Mota ELA, Macinko J, et al. Principais causas de internação por condições sensíveis à atenção primária no Brasil: uma análise por faixa etária e região. Rev Bras Saúde Mater Infant. 2010 nov;10(supl 1):83-91.

27. Ministério da Saúde (BR). Departamento de Informática do Sistema Único de Saúde DATASUS. Pesquisa nacional de saúde 2013: módulo de utilização de serviços de saúde [Internet]. 2013 [citado 2016 jul 25]. Disponível em: http://tabnet.datasus.gov.br/cgi/deftohtm. exe?pns/pnsj.def

28. Bittencourt AS, Camacho LAB, Leal MC. 0 sistema de informação hospitalar e sua aplicação na saúde coletiva. Cad Saúde Pública. 2006 jan;22(1):19-30.

29. Abaid RA, Nedel FB, Alcayaga EL. Condições sensíveis à atenção primária: Confiabilidade diagnóstica em Santa Cruz do Sul, RS. Rev Epidemiol Control Infect. 2014 jul;4(3):208-14.

30. Caminal-Homar J, Casanova-Matutano C. La evaluación de la atención primaria y las hospitalizaciones por ambulatory care sensitive conditions. Marco conceitual. Aten Primaria. 2003 ene;31(1):61-5. 


\begin{abstract}
Objective: to describe proportions and rates of hospitalization for Ambulatory Care Sensitive Conditions (ACSC) among children under 5 years old, in Santa Catarina State, Brazil, 2012. Methods: this is a descriptive study using Brazilian National Health Service Hospital Information System (SIH/SUS) data, classified according to the Brazilian ACSC List. Results: a total of 32,445 children aged $<5$ years old were hospitalized, $25,7 \%$ of whom were ACSC cases, representing a rate of 20.1/1,000 inhabitants in the same age group; the main causes were infectious gastroenteritis and complications (26.7\%), bacterial pneumonia (22.2\%) and pulmonary diseases (16.9\%); there was a higher hospitalization rate due to ACSC in males $(21.1 / 1,000)$, and in children $<1$ year old $(43.8 / 1,000)$. Conclusion: The results showed that the State of Santa Catarina had lower rates and proportions than those found in other Brazilian studies, even though ACSC were the cause of one quarter of hospitalizations in children aged $<5$ years.
\end{abstract}

Keywords: Primary Health Care; Epidemiology, Descriptive; Hospitalization; Pediatrics; Health Status Indicators.

\section{Resumen}

Objetivo: describir proporciones y tasas de hospitalización por Condiciones Sensibles a la Atención Primaria (CSAP) en menores de 5 años de edad en el estado de Santa Catarina, Brasil, en 2012. Métodos: estudio descriptivo, utilizando las bases del Sistema de Informaciones Hospitalarias del Sistema de Salud Brasileño (SIH/SUS), clasificadas de acuerdo a la Lista Brasileña de CSAP. Resultados: se registraron 32.445 hospitalizaciones en menores de 5 años; $25,7 \%$ de ellas representadas por las CSAP, con una tasa de 20,1/1.000 babitantes del mismo grupo de edad; las principales causas fueron gastroenteritis infecciosas y complicaciones (26,7\%), neumonías bacterianas (22,2\%) y enfermedades pulmonares (16,9\%); se observó una mayor tasa de bospitalización por CSAP en el sexo masculino $(21,1 / 1.000)$ y en $<1$ año de edad (43,8/1.000). Conclusión: los resultados evidenciaron que el estado de Santa Catarina presentó tasas y proporciones menores que las observadas en estudios brasileños, aunque las CSAP hayan sido la causa de 1/4 de las internaciones.

Palabras-clave: Atención Primaria de Salud; Epidemiologia descriptiva; Hospitalización; Pediatría; Indicadores de Salud.

Recebido em 13/09/2017

Aprovado em 21/12/2017 